\title{
DIVISION I / WORKING GROUP ASTROMETRY BY SMALL GROUND-BASED TELESCOPES
}

\author{
CHAIR \\ VICE-CHAIR \\ MEMBERS
}

\author{
William Thuillot \\ Magdalena Stavinschi \\ Alexander H. Andrei, Jean-Eudes Arlot, \\ Marcelo Assafin, N. Bazey, \\ George A. Gontcharov, Rustem Gumerov, \\ Jin Wenjing, Jose L. Muinos Haro, \\ Panayiotis Niarchos, Jose Pereira Osório, \\ Dan Pascu, Thierry Pauwels, \\ Gennadiy I. Pinigin, Y. Prostyuk, \\ A. Pugliano, Theodore J. Rafferty, \\ Jane L. Russell, Vladimir V. Rylkov, \\ M. Luisa Sanchez, Alexander A. Shulga, \\ Jean Souchay, Zhenghong Tang, \\ Ramachrisna Teixeira, Arthur R. Upgren, \\ William F. van Altena, Roberto Vieira Martins \\ Norbert Zacharias
}

\section{TRIENNIAL REPORT 2006 - 2009}

\section{Introduction}

At the IAU XXVI General Assembly in 2006, the Division I decided to create the Working Group on Astrometry by Small Ground-Based Telescopes (WG-ASGBT). Its scientic goals are to foster the follow-up of small bodies detected by the large surveys including the NEOs; to set-up a dedicated observation network for the follow-up of objects which will be detected by Gaia; to contribute to the observation campaigns of the mutual events of natural satellites, stellar occultations, and binary asteroids; and to encourage teaching astrometry for the next generation. The present report gives the main activities carried out in these areas with small telescopes (diameter less than $2 \mathrm{~m}$ ).

\section{Research developments within the past triennium}

Several members of the working group report on recent activities in astrometry by small ground-based telescopes carried out by themselves or their team. The Working Group has presented a part of these activities during IAU Symposium No. 248 in Shanghai in 2007 (Thuillot et al. 2008).

N. Zacharias (USNO, Washington, USA) reports that the Northern and Southern Proper Motion (NPM, SPM) data (Lick Observatories, Yale/San-Juan) were re-reduced in a joined Yale/USNO effort. The UCAC3 is completed for a 2009 release of higly accurate positions and proper motions of about 80 million stars. The $20 \mathrm{~cm}$ USNO 'red lens' astrograph has been refurbished for a new all-sky observing program with a 440 
million pixel camera. This 'U-mouse' astrometric survey will begin in 2009 from Cerro Tololo. For more details please see the IAU WG on Densification of the Reference Frame triennial report in this volume and Zacharias (2008, in IAU Symposium No 248).

Wenjing Jin (Shanghai Astronomical Observatory, China) reports on various astrometric activities in China. The peculiar following works were published. The 1.0/1.2 m NEOST (Near Earth Objects Space Telescope) equipped with a $4096 \times 4096$ SI CCD detector was installed completely and began to test observation. More than 188 new asteroids have been found including an Apollo-type NEO-2007 JW2. A new Jupiter-family comet, P/2007 S1, was discovered (Zhao et al. 2008).

A collaboration with Nikolaev Astronomical Observatory (NAO), Ukraine, used the $25 \mathrm{~cm}$ telescope of Shanghai Astronomical Observatory equipped with a CCD camera S1C-077 in drift scan mode. Observations for GSS (Geo-Stationary Satellite) were carried out (Mao et al. 2007).

Astrometric observations of Triton have been done at the Shanghai $1.56 \mathrm{~m}$ telescope. Comparison with the theoretical positions provided from JPL and IMCCE showed a standard deviation of $0^{\prime \prime} .04$ of the O-C (Qiao et al. 2006).

Astrometric observations of Phoebe has been done at the $1 \mathrm{~m}$ telescope of the Yunnan Observatory and analyzed. Comparison the JPL ephemerides DE405 and SAT199 showed mean residuals of 210 mas and -50 mas in right ascension and declination, respectively, and a standard deviation of 6 mas (Peng \& Zhang 2006).

Observations of the same satellite has been done at the Shanghai $1.56 \mathrm{~m}$ telescope. Comparison with JPL ephemerides show standard deviations in right ascension and declination are 58 mas and 7 mas respectively (Qiao et al. 2007).

Mutual events of the Galilean satellites has been observed with the $1 \mathrm{~m}$ telescope at Yunnan Observatory and analyzed. Astrometric positions were deduced and the comparisons were made with the theoretical models, Lieske's E5 and Lainey's L1. The accuracy of positions in RA and DEC are 103 and 88 mas for Lieske's theory while 74 and 80 mas for Lainey's theory (Peng \& Noyelles 2007; Peng et al. 2008).

At IMCCE, Paris Observatory, France, improvement of the dynamical models and researches for the detection of small suspected effects (tidal effects, effect of the Yarkovsky force, ...) on the motion of the Solar System bodies are performed. Highly accurate observational data are required and small telescopes are generally well adapted for this goal. Mutual events of the Uranian satellites, which occur every $42 \mathrm{yr}$ and had never been observed until this occurrence, were predicted by (Arlot et al. 2006) and observed through an international campaign. The analysis is still in progress, only preliminary results are published at that time (Birlan et al. 2008).

As a complement of a program of detection of satellites of asteroids by large telescopes, observations by small telescopes are very useful such as those of mutual events. Such events have been predicted, surveyed and observed thanks to international campaigns: Antiope in 2005 (Descamps et al. 2007a), Patroclus in 2006 and 2007 (Berthier et al. 2007), Kalliope in 2007 (Descamps et al. 2007b). Orbital parameters and morphological characterization of these systems have been deduced.

An analysis of observations of planets and natural satellites performed at the meridian transit circle of Bordeaux Observatory, France, during the period 1997-2007 has been done. The observations of the planetary satellites provide pseudo observations of the planet itself. The results show the interest of continuing this type of observation (Arlot et al. 2008).

Researchers of IMCCE are involved in the preparation of the Gaia mission for the program related to the Solar System objects. A ground-based network of observers is 
being organized in order to perform follow-up observations of the Solar System bodies which could be detected by Gaia but difficult or impossible to re-observe by the satellite itself (Mignard et al. 2007).

M. Assafin (Obs. di Valongo, Rio de Janeiro, Brazil) reports on the activity of the Brazilian astrometric group. Astrometry of natural satellites is performed at the $1.6 \mathrm{~m}$ and $0.6 \mathrm{~m}$ of the Itajuba National Observatory, in particular for Jupiter (Elara, Himalia, Pasiphae, Carme), Saturn (Titan, Phoebe, Iapetus, Hiperion), Uranus (all main five ones), Neptune (Triton, Nereid). This group gives also an important contribution to the international campaigns of observation of the mutual events of the satellites lead by IMCCE, and recently for the Uranian satellites. It is also strongly involved in the campaign of observation of the mutual events of the binary asteroids (Descamps et al. 2007a).

Through a collaboration with B. Sicardy (Observatoire de Paris) predictions of stellar occultations by Pluto, TNOs or natural satellites are periodically made on the basis of the Brazilian astrometic observations. The improvement of these predictions thanks to last minute astrometry helps to choose the location of the mobile telescopes. The occultation event by Pluto on 14 July 2007 was successfuly observed on the $1.6 \mathrm{~m}$ telescope by this group (Sicardy et al. 2007). In a collaboration with astronomers from the Bucharest astronomical institute, Romania, the observations of ICRF sources have been performed at the $0.6 \mathrm{~m}$ Zeiss telescope at Belogradchik Observatory, Bulgaria (Assafin et al. 2007).

Th. Pauwels (ROB, Brussels, Belgium) describes the activity of the astrometry group of the Royal Observatory of Belgium. It performs several observations at the Uccle Schmidt telescope ( $0.85 \mathrm{~cm}$ aperture, $2 \mathrm{~m}$ focal length): 6687 positions of asteroids and 16 positions of comets were published in the MPCs (Observers P. De Cat, H. Debehogne, R. Desmet, E. Elst, A. Jonckheere, T. Pauwels, K. Puttemans). 41 positions of asteroids and 16 positions of comets were published in the MPECs (Observers P. De Cat and T. Pauwels). 28 occultations of stars by minor planets were attempted, of which 1 positive event. 8 light curves were obtained of mutual occultations and eclipses of an asteroid and its satellite (Berthier et al. 2007).

G. Pinigin, in collaboration with A. Shulga, A. Ivantsov and L.Hudkova, reports on observations and analysis done at the Research Institute National Astronomical Observatory (RI NAO, Nikolaev, Ukraine) with robotic or automatic telescopes. Fast Robotic Telescope FRT ( $D=300 \mathrm{~mm}, F=1500 \mathrm{~mm}$ )in a multichannel mode with rotary platform is used for observations of NEAs, artificial satellites and space debris in all orbits. The Axial Meridian Cirle AMC $(D=180 \mathrm{~mm}, F=2480 \mathrm{~mm})$, a modern CCD robotic telescope, has been upgraded with the CCD computer control and remote access. It is used for observations of stars and small Solar System bodies up to 17.5 mag. The telescope AZT-8 $(\mathrm{D}=700 \mathrm{~mm}, \mathrm{~F}=285 \mathrm{~mm})$ is used for CCD astronomical research of objects up to $19 \mathrm{mag}$. in the near-Earth space since 2006 (agreement between Nat. Cent. Space Control and RI NAO).

G. Pinigin also reports on the international joint project between Turkish National Observatory TUBITAK (Z. Aslan), Kazan University, Russia (R. Gumerov) and NAO (Ukraine) on the observations of the small Solar System bodies at the TUBITAK RTT150 telescope $(D=1500 \mathrm{~mm}, \mathrm{~F} / 8)$. The positional and photometric observations of asteroids down to $20 \mathrm{mag}$ were made there in 2004-2008, and part of them was sent to the MPC. According to the astrometric precision obtained (50 mas), this telescope is well adapted for the Gaia follow-up program. New masses for 21 asteroids were determined by the dynamical method using modern ground-based CCD observations of the selected 
asteroids and dynamical model of asteroid motions, based on the DE405 (Aslan et al. 2007, Ivantsov 2007).

CCD observations of 200 fields around extragaclactic radiosources obtained in 2000 2003 with the RTT150 (TUG, Turkey) and $1 \mathrm{~m}$ telescope of Yunnan Observatory (Shanghai Astronomical Observatory, China) were used for collaborative research on refinement of linking optical-radio reference frames in Nikolaev with the reference 2MASS catalog.

W. van Altena (Yale Univ., New Haven, USA) reports on the second-epoch observations for the Yale/San-Juan Southern Proper Motion (SPM) program which will consist of approximately 100 million absolute proper motions from magnitude 5 to 17.5 , magnitudes and colors in the blue and visual passbands. CCD frames are obtained with the $51 \mathrm{~cm}$ double astrograph at Cesco Observatory in El Leoncito, Argentina. Sky coverage south of -20 degrees declination is essentially complete as of June 2008.

Proper-motion determinations for six globular clusters (Casetti-Dinescu et al. 2007) provided a testbed for the combination of the first-epoch plate material with secondepoch CCD astrometry. Work is well underway on the next SPM catalog that will cover the sky south of -20 degrees. It is anticipated that the SPM4 will contain roughly 100 million stars and galaxies and be more than 90 percent complete to $V=17.5$.

\section{Education}

Thanks to a joint initiative by astronomers from France (IMCCE) and China (Shanhai observatory, Beijing Planetarium, Jinan University of Guangzhou), a first Chinese-French Spring School on Astrometry 'observational campaign of solar system bodies', was held in Beijing, 7-13 April, 2008. The purpose was to foster students and young researchers to do researches in astrometry and to study new astrometric methods. 28 students and young astronomers attended this spring school. The lectures were given on fundamental astrometry, receptors, telescopes and images for astrometric purpose, astrometry through photometry and observational campaigns for phenomena, practical astrometry, space astrometry. In 2008, a census is organized by the working group in order to identify the universities and observatories where astrometry is teached and to know the topics of the main cursus delivered there. The results will be posted on the working group web page <www.imcce.fr/astrom/>.

William Thuillot chair of the Working Group

\section{References}

Arlot, J.-E., Lainey, V., \& Thuillot, W. 2006, A\&A, 456, 1173

Arlot, J.-E., Dourneau, G., \& Lecampion, J.-F. 2007, $A \& A, 484,869$

Aslan, Z., Gumerov, R., et al. 2007, in: O. Demircan, S. O. Selam \& B. Albayrak (eds.), Solar and Stellar Physics Through Eclipses, ASP-CS, 370, 52

Assafin, M., Nedelcu, D. A., Popescu, P., et al. 2007, A\&GA, 476, 989

Berthier, J., Marchis, F., et al. 2007, AAS, Proc. DPS, 39, 35.05

Birlan, M., Nedelcu, D. A., et al. 2008, Astron. Nach., 329, 567

Casetti-Dinescu, D. I., Girard, T. M., Herrera, D., et al. 2007, AJ, 134, 195

Descamps, P., Marchis, F., Michalowski, T., et al. 2007, Icarus, 187, 482

Descamps, P., Marchis, F., Pollock, J., et al. 2007, P\&SSS, 56, 1851

Ivantsov, A. V. 2007, Kinematics and Physics of Celestial Bodies, 23, 3, 108

Mao, Y. D., Tang, Z. H., Tao Jun, et al. 2007, Acta Astronomica Sinica, Vol. 48, No. 4, 475 
Mignard, F., Cellino, A., Muinonen, K. et al. 2007, EMESP, 101, 97

Peng, Q. Y. \& Zhang, Q. F. 2006, MNRAS, 366, 208

Peng, Q. Y. \& Noyelles, B. 2007, ChJAA, Vol. 7, No. 2, 317

Peng, Q. Y., Emelyanov, N. V., et al. 2008, in: W. J. Jin, I. Platais \& M. A. C. Perryman (eds.), A Giant Step: from Milli- to Micro-arcsecond Astrometry, Proc. IAU Symp. No. 248 (Cambridge: CUP), p. 114

Qiao, R. C., Tang, Z. H, Shen, K. X., Dourneau, G., et al. 2006, A\& A, 454, 379

Qiao, R. C., Yan, Y. R., Shen, K. X., et al. 2007, MNRAS, 376, 1707

Sicardy, B., Widemann, T. et al. 2007, AAS, Proc. DPS, 39, 62.02

Thuillot, W., Stavinschi, M., Assafin, M., \& the ABSGBT working group 2008, in: W. J. Jin, I. Platais \& M. A. C. Perryman (eds.), A Giant Step: from Milli- to Micro-arcsecond Astrometry, Proc. IAU Symposium No. 248 (Cambridge: CUP), p. 286

Zhao, H. B.,Yao, J. S, \& Lu, H. 2008, in: W. J. Jin, I. Platais \& M. A. C. Perryman (eds.), A Giant Step: from Milli- to Micro-arcsecond Astrometry, Proc. IAU Symposium No. 248 (Cambridge: CUP), p. 505 